\title{
Theory of Non-Hermitian Fermionic Superfluidity with a Complex-Valued Interaction
}

\author{
Kazuki Yamamoto, ${ }^{1, *}$ Masaya Nakagawa, ${ }^{2,3}$ Kyosuke Adachi, ${ }^{1,4}$ Kazuaki Takasan, ${ }^{1,5}$ \\ Masahito Ueda, ${ }^{3,2}$ and Norio Kawakami ${ }^{1}$ \\ ${ }^{1}$ Department of Physics, Kyoto University, Kyoto 606-8502, Japan \\ ${ }^{2}$ RIKEN Center for Emergent Matter Science (CEMS), Wako, Saitama 351-0198, Japan \\ ${ }^{3}$ Department of Physics, University of Tokyo, 7-3-1 Hongo, Bunkyo-ku, Tokyo 113-0033, Japan \\ ${ }^{4}$ RIKEN Center for Biosystems Dynamics Research (BDR), Kobe, Hyogo, 650-0047, Japan \\ ${ }^{5}$ Department of Physics, University of California, Berkeley, California 94720, USA
}

(Received 11 March 2019; published 16 September 2019)

\begin{abstract}
Motivated by recent experimental advances in ultracold atoms, we analyze a non-Hermitian (NH) BCS Hamiltonian with a complex-valued interaction arising from inelastic scattering between fermions. We develop a mean-field theory to obtain a $\mathrm{NH}$ gap equation for order parameters, which are different from the standard BCS ones due to the inequivalence of left and right eigenstates in the NH physics. We find unconventional phase transitions unique to $\mathrm{NH}$ systems: superfluidity shows reentrant behavior with increasing dissipation, as a consequence of nondiagonalizable exceptional points, lines, and surfaces in the quasiparticle Hamiltonian for weak attractive interactions. For strong attractive interactions, the superfluid gap never collapses but is enhanced by dissipation due to an interplay between the BCS-BEC crossover and the quantum Zeno effect. Our results lay the groundwork for studies of fermionic superfluidity subject to inelastic collisions.
\end{abstract}

DOI: 10.1103/PhysRevLett.123.123601

Introduction.-In recent years, non-Hermitian (NH) quantum systems have been actively studied both experimentally and theoretically [1-36]. NH quantum systems arise when the system undergoes dissipation to an environment $[37,38]$. It has been revealed that non-Hermiticity drastically alters the properties of a number of quantum phenomena that have been established in the Hermitian physics, such as quantum phase transitions [1-4], quantum critical behavior [5-7], topological phases [8-17], and magnetism [18]. Such theoretical predictions have been confirmed experimentally by using optical systems and ultracold atoms [22-30]. However, since most of the previous studies dealt with single-particle physics, exploration of many-body physics in $\mathrm{NH}$ systems is still in its infancy [3-6,31-34].

Fermionic superfuidity is one of the most striking quantum many-body phenomena, which has been a subject of intensive investigation in condensed matter physics [39]. More recently, ultracold atomic systems have opened a new arena to study fermionic superfluidity [40-42], where they are subject to losses due to inelastic collisions. For example, if we consider a superfluid mediated by the orbital Feshbach resonance [43-46], which controls an interaction between the ground state and an excited state of an atom [47-50], loss inevitably occurs due to inelastic processes between different orbitals. Such inelastic two-body losses cause the decay of eigenstates of the Hamiltonian and may be described by complex-valued interactions, thus providing an ideal playground to study NH fermionic superfluids. Despite its growing importance, however, theory for $\mathrm{NH}$ fermionic superfluidity has not been established yet [19-21].

In this Letter, we demonstrate how fermionic superfluidity in ultracold atoms is modified under inelastic collisions, by generalizing the standard BCS theory to a situation in which fermions interact with each other via a complex-valued attraction. We elucidate that the nonHermiticity alters several fundamental properties of superfluidity; for example, the order parameters of particles and holes are not necessarily complex conjugate to each other in the $\mathrm{NH}$ physics, and the Bogoliubov quasiparticles obey neither Fermi nor Bose statistics since eigenstates are, in general, not orthogonal to each other.

Furthermore, we find that the non-Hermiticity leads to unique quantum phase transitions in superfluids. For a weak interaction, the real part of the superfluid gap is first suppressed and then quenched with increasing dissipation. Remarkably, superfluidity is restored beyond a certain strength of dissipation and the superfluid gap is even enhanced afterwards with increasing dissipation. We show that these phase transitions emerge from exceptional points, lines and surfaces that are unique to the $\mathrm{NH}$ physics, where the Hamiltonian cannot be diagonalized [51,52]. For a strong interaction, superfluidity is not suppressed and never breaks down because fermions are paired to form molecules on each site, thereby avoiding intersite decoherence. Our finding can experimentally be tested in various ultracold atomic species under inelastic collisions such as ${ }^{173} \mathrm{Yb}$, ${ }^{40} \mathrm{~K}$, and ${ }^{6} \mathrm{Li}[49,50,53-57]$. 
Model.-We consider ultracold fermionic atoms with an attractive interaction in a three-dimensional optical lattice. When atoms undergo inelastic collisions, the scattered atoms are lost from the system since a large internal energy is converted to the kinetic energy. An atomic gas undergoing two-body losses due to inelastic collisions is described by a quantum master equation [37]

$$
\begin{aligned}
\dot{\rho} & =-i[H, \rho]-\frac{1}{2} \gamma \sum_{i}\left(L_{i}^{\dagger} L_{i} \rho+\rho L_{i}^{\dagger} L_{i}-2 L_{i} \rho L_{i}^{\dagger}\right) \\
& =-i\left(H_{\mathrm{eff}} \rho-\rho H_{\mathrm{eff}}^{\dagger}\right)+\gamma \sum_{i} L_{i} \rho L_{i}^{\dagger},
\end{aligned}
$$

where $L_{i}$ is a Lindblad operator that describes a loss at site $i$ with rate $\gamma$, and $\rho$ is the density matrix of the atomic gas. When the quantum-jump term, which is the last term in Eq. (1), is negligible, the system is described by an effective NH Hamiltonian $H_{\text {eff }}=H-(i / 2) \gamma \sum_{i} L_{i}^{\dagger} L_{i}$. Such a situation is realized when we consider the dynamics over a sufficiently short time compared with the inverse loss rate $1 / \gamma$ [31], which characterizes the timescale where the effect of quantum jumps becomes significant. In this case, the lowest real part of the eigenspectrum gives the effective ground state, and the imaginary part of energy corresponds to a decay rate of each eigenstate. The two-body loss is described by $L_{i}=c_{i \downarrow} c_{i \uparrow}$, giving a NH BCS Hamiltonian

$$
H_{\mathrm{eff}}=\sum_{k \sigma} \xi_{k} c_{k \sigma}^{\dagger} c_{k \sigma}-U \sum_{i} c_{i \uparrow}^{\dagger} c_{i \downarrow}^{\dagger} c_{i \downarrow} c_{i \uparrow},
$$

with a complex-valued interaction $U=U_{1}+i \gamma / 2$, where $U_{1}, \gamma>0$. Here, $\xi_{k} \equiv \epsilon_{k}-\mu, \epsilon_{k}$ is the energy dispersion, $\mu$ is the chemical potential, and $c_{k \sigma}$ and $c_{i \sigma}$ denote annihilation operators of a spin- $\sigma$ fermion with momentum $\boldsymbol{k}$ and at site $i$, respectively. In this Letter, we formulate a meanfield theory from $H_{\text {eff }}$ and elucidate how unconventional properties of superfluidity emerge in NH BCS systems.

Formulation of the NH mean-field theory.-We first clarify how the standard BCS mean-field theory is changed due to non-Hermiticity by formulating it with a pathintegral approach. We start with a partition function defined as

$$
Z=\sum_{n} e^{-\beta E_{n}}=\sum_{n}\left\langle E_{n}\left|e^{-\beta H_{\mathrm{eff}}}\right| E_{n}\right\rangle_{R} .
$$

Here, ${ }_{L}\left\langle E_{n}\right|$ and $\left|E_{n}\right\rangle_{R}$ are left and right eigenstates of $H_{\text {eff }}$ with eigenenergy $E_{n}$, and they satisfy an orthonormal relation ${ }_{L}\left\langle E_{n} \mid E_{m}\right\rangle_{R}=\delta_{n m}$ [58]. We note that, as temperature is not well defined in generic open quantum systems, we only consider the infinite limit of $\beta$ to elucidate the physics of the ground state and calculate the excitation spectrum. Thus, $\beta$ is a parameter used to formulate a path integral and should not be regarded as the temperature of the system. We use a path-integral representation of the partition function [Eq. (3)] to perform the HubbardStratonovich transformation with auxiliary fields $\Delta, \bar{\Delta}$ and then integrate out the fermionic degrees of freedom to obtain $Z=\int \mathcal{D} \bar{\Delta} \mathcal{D} \Delta e^{-S_{\text {eff }}(\Delta, \bar{\Delta})}$, where $S_{\text {eff }}$ is the effective action given by [59]

$$
S_{\text {eff }}(\bar{\Delta}, \Delta)=-\sum_{\omega_{n}, k} \log \left(\omega_{n}^{2}+\xi_{k}^{2}+\bar{\Delta} \Delta\right)+\frac{\beta N}{U} \bar{\Delta} \Delta .
$$

Here, $N$ denotes the number of lattice sites, and $\omega_{n}$ is the Matsubara frequency of fermions. The saddle point condition for the partition function, $\partial S_{\mathrm{eff}} / \partial \Delta=\partial S_{\mathrm{eff}} / \partial \bar{\Delta}=0$, yields the $\mathrm{NH}$ gap equation

$$
\frac{N}{U}=\sum_{k} \frac{1}{2 \sqrt{\xi_{k}^{2}+\bar{\Delta} \Delta}} \tanh \frac{\beta \sqrt{\xi_{k}^{2}+\bar{\Delta} \Delta}}{2},
$$

if there exists a nontrivial solution other than $\Delta=\bar{\Delta}=0$. We set $\beta$ to infinity to obtain an effective ground state. The chemical potential $\mu$ is determined so that the mean particle number in the non-Hermitian ensemble [Eq. (3)] is equal to the particle number of the density-matrix sector of interest [59].

In NH physics, four distinct types of order parameters can be defined according to whether left and right eigenstates are assigned to the bra or ket vectors in the expectation value. Importantly, the expectation value of an operator $A$ calculated from the non-Hermitian ensemble [Eq. (3)] should correspond to ${ }_{L}\langle A\rangle_{R} \equiv \sum_{n L}\left\langle E_{n}|A| E_{n}\right\rangle_{R} e^{-\beta E_{n}} / Z$. Thus, the order parameters corresponding to the superfluid gap are given by [59]

$$
\begin{aligned}
& \Delta=-\frac{U}{N} \sum_{k}{ }_{L}\left\langle c_{-k \downarrow} c_{k \uparrow}\right\rangle_{R}, \\
& \bar{\Delta}=-\frac{U}{N} \sum_{k}\left\langle c_{k \uparrow}^{\dagger} c_{-k \downarrow}^{\dagger}\right\rangle_{R},
\end{aligned}
$$

indicating that $\bar{\Delta} \neq \Delta^{*}$ since $\left|E_{n}\right\rangle_{L} \neq\left|E_{n}\right\rangle_{R}$. As discussed below, this leads to various intriguing consequences on the properties of a NH superfluid.

To elucidate the effect of non-Hermiticity, let us apply the mean-field decoupling to the NH BCS Hamiltonian with the simplest $s$-wave pairing interaction

$$
H_{\text {eff }}=\sum_{k \sigma} \xi_{k} c_{k \sigma}^{\dagger} c_{k \sigma}-\frac{U}{N} \sum_{k k^{\prime}} c_{k \uparrow}^{\dagger} c_{-k \downarrow}^{\dagger} c_{-k^{\prime} \downarrow} c_{\boldsymbol{k}^{\prime} \uparrow} \cdot
$$

Substituting $\quad c_{\boldsymbol{k} \uparrow}^{\dagger} c_{-\boldsymbol{k} \downarrow}^{\dagger}={ }_{L}\left\langle c_{\boldsymbol{k} \uparrow}^{\dagger} c_{-\boldsymbol{k} \downarrow}^{\dagger}\right\rangle_{R}+\delta\left(c_{\boldsymbol{k} \uparrow}^{\dagger} c_{-\boldsymbol{k} \downarrow}^{\dagger}\right) \quad$ and $c_{-k \downarrow} c_{k \uparrow}={ }_{L}\left\langle c_{-k \downarrow} c_{k \uparrow}\right\rangle_{R}+\delta\left(c_{-k \downarrow} c_{k \uparrow}\right)$ into Eq. (8) and neglecting the second-order terms in $\delta$, we obtain the mean-field Hamiltonian 


$$
H_{\mathrm{MF}}=\sum_{k}\left(\begin{array}{cc}
c_{\boldsymbol{k} \uparrow}^{\dagger} & c_{-k \downarrow}
\end{array}\right)\left(\begin{array}{cc}
\xi_{\boldsymbol{k}} & \Delta \\
\bar{\Delta} & -\xi_{k}
\end{array}\right)\left(\begin{array}{c}
c_{\boldsymbol{k} \uparrow} \\
c_{-\boldsymbol{k} \downarrow}^{\dagger}
\end{array}\right),
$$

which is diagonalized as $H_{\mathrm{MF}}=\sum_{k} E_{\boldsymbol{k}}\left(\bar{\gamma}_{\boldsymbol{k} \uparrow} \gamma_{\boldsymbol{k} \uparrow}+\bar{\gamma}_{-\boldsymbol{k} \downarrow} \gamma_{-\boldsymbol{k} \downarrow}\right)-$ $\sum_{k} E_{k}$. The quasiparticle operators $\bar{\gamma}_{k \sigma}, \gamma_{k \sigma}$ and the corresponding energy $E_{k}$ are given by $\bar{\gamma}_{k \uparrow}=u_{k} c_{k \uparrow}^{\dagger}-\bar{v}_{k} c_{-k \downarrow}$, $\gamma_{k \uparrow}=u_{k} c_{k \uparrow}-v_{k} c_{-k \downarrow}^{\dagger}$ (and similar equations hold for $\bar{\gamma}_{k \downarrow}$, $\left.\gamma_{k \downarrow}\right)$ and $E_{k}=\sqrt{\xi_{k}^{2}+\bar{\Delta} \Delta}$, respectively, where the coefficients satisfy $u_{k}^{2}+v_{k} \bar{v}_{k}=1$ (for their explicit forms, see the Supplemental Material [59]). In the Hermitian limit, $\bar{\gamma}_{\boldsymbol{k} \sigma}$ and $\bar{v}_{\boldsymbol{k}}$ respectively reduce to $\gamma_{\boldsymbol{k} \sigma}^{\dagger}$ and $v_{\boldsymbol{k}}^{*}$, which describe the Bogoliubov quaiparticles. Here, we note that the mean-field Hamiltonian is non-Hermitian since $\Delta^{*} \neq \bar{\Delta}$, and the quasiparticle operators $\gamma_{k \sigma}$ and $\bar{\gamma}_{k \sigma}$ are not Hermitian conjugate to each other. Therefore, the Hamiltonian cannot be diagonalized via a unitary transformation.

As a result, the right and left ground states are defined by $\gamma_{\boldsymbol{k} \sigma}|\mathrm{BCS}\rangle_{R}=0$ and $\bar{\gamma}_{\boldsymbol{k} \sigma}^{\dagger}|\mathrm{BCS}\rangle_{L}=0$, respectively, where

$$
\begin{aligned}
& |\mathrm{BCS}\rangle_{R}=\prod_{\boldsymbol{k}}\left(u_{\boldsymbol{k}}+v_{\boldsymbol{k}} c_{\boldsymbol{k} \uparrow}^{\dagger} c_{-\boldsymbol{k} \downarrow}^{\dagger}\right)|0\rangle, \\
& |\mathrm{BCS}\rangle_{L}=\prod_{\boldsymbol{k}}\left(u_{\boldsymbol{k}}^{*}+\bar{v}_{\boldsymbol{k}}^{*} c_{\boldsymbol{k} \uparrow}^{\dagger} c_{-k \downarrow}^{\dagger}\right)|0\rangle,
\end{aligned}
$$

and $|0\rangle$ is the vacuum for fermions. They satisfy ${ }_{L}\langle\mathrm{BCS} \mid \mathrm{BCS}\rangle_{R}=1$ and reproduce the ordinary BCS ground state in the Hermitian limit. We thus obtain $H \bar{\gamma}_{k \sigma}|\mathrm{BCS}\rangle_{R}=$ $E_{\boldsymbol{k}} \bar{\gamma}_{\boldsymbol{k} \sigma}|\mathrm{BCS}\rangle_{R}$ and $H^{\dagger} \gamma_{k \sigma}^{\dagger}|\mathrm{BCS}\rangle_{L}=E_{\boldsymbol{k}}^{*} \gamma_{k \sigma}^{\dagger}|\mathrm{BCS}\rangle_{L}$, which imply that $\bar{\gamma}_{k \sigma}$ and $\gamma_{k \sigma}^{\dagger}$ create the right and left eigenstates, respectively, when acted on the ground state. Here, we have shifted the ground state energy to zero. Using Eqs. (6), (7), (10), and (11), we obtain the $\beta \rightarrow \infty$ limit of the NH gap equation as $N / U=\sum_{k} 1 / 2 E_{k}$, which is solved selfconsistently. We note that the quasiparticle operators satisfy an anticommutation relation $\left\{\gamma_{k \sigma}, \bar{\gamma}_{k^{\prime} \sigma^{\prime}}\right\}=\delta_{k k^{\prime}} \delta_{\sigma \sigma^{\prime}}$, although these quasiparticles obey neither Fermi nor Bose statistics due to $\gamma_{k \sigma}^{\dagger} \neq \bar{\gamma}_{k \sigma}$, reflecting non-Hermiticity of the meanfield Hamiltonian.

Here, we point out an important relation between the order parameters $\Delta$ and $\bar{\Delta}$. In the Hermitian case, they are complex conjugate to each other and we can choose a gauge where $\Delta$ is real without loss of generality. This is equivalent to requiring $H_{\mathrm{MF}}^{\dagger}=H_{\mathrm{MF}}^{*}$ in the matrix representation in the Fock-state basis in the Hilbert space. Now we consider the $\mathrm{NH}$ case. As in the Hermitian case, the $\mathrm{NH}$ BCS Hamiltonian [Eq. (8)] satisfies a symmetry relation $H^{\dagger}=H^{*}$ under the matrix representation in terms of Fock states, indicating that the left eigenstates are obtained through complex conjugation of the right ones. The $\mathrm{NH}$ BCS Hamiltonian has the U(1) symmetry as in the Hermitian case and this is not affected by the complex nature of the interaction. Then, when a superfluid is formed, its ground states become degenerate due to spontaneous U(1) symmetry breaking. The BCS ground states [Eqs. (10) and (11)] are consistent with these properties if

$$
\begin{aligned}
& \Delta(\theta)=\Delta_{0} e^{i \theta}, \\
& \bar{\Delta}(\theta)=\Delta_{0} e^{-i \theta},
\end{aligned}
$$

where $\Delta_{0} \in \mathbb{C}$ and $\theta$ is the $\mathrm{U}(1)$ phase. By choosing a special gauge for which $H_{\mathrm{MF}}^{\dagger}=H_{\mathrm{MF}}^{*}$ is satisfied, we have $\Delta=\bar{\Delta}$. Here, we note that the relation [Eq. (12)] is specific to the NH BCS Hamiltonian [Eq. (8)] and may be changed depending on symmetry of a NH Hamiltonian.

Quantum phase transitions of a NH superfluid.-We solve the gap equation at $\beta \rightarrow \infty$ numerically (for analytic solutions in the case of a constant density of states, see the Supplemental Material [59]). Figure 1 shows the superfluid order parameter $\Delta_{0}$. Here, for simplicity, we consider a system with particle-hole symmetry, and set the chemical potential measured from the Fermi energy to zero [59]. For small $U_{1}, \operatorname{Re} \Delta_{0}$ is suppressed by dissipation $\gamma$ and then vanishes, indicating a breakdown of superfluidity. Remarkably, as $\gamma$ increases, the superfluid solution reappears, and the gap size $\operatorname{Re} \Delta_{0}$ is enhanced due to dissipation, eventually exceeding the value in the Hermitian limit. On the other hand, for strong attractive interaction, $\operatorname{Re} \Delta_{0}$ is not suppressed, but rather enhanced due to dissipation.

The qualitative difference between the cases of weak and strong attractions is explained by an interplay between the BCS-BEC crossover [62-65] and dissipation. In the strongdissipation limit, the behavior of the system is governed by the continuous quantum Zeno effect (QZE) [66-71], which suppresses tunneling to neighboring sites, leading to localization of particles. In the case of weak attraction, the intersite coherence of Cooper pairs is suppressed by dissipation and the superfluidity is destroyed. However,
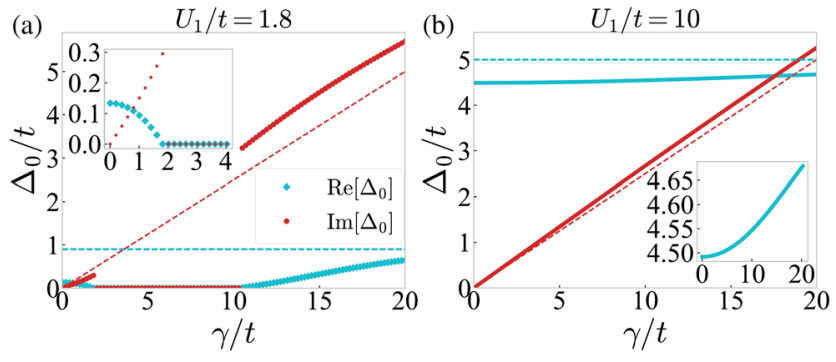

FIG. 1. Numerical solution of $\Delta_{0}$ as a function of $\gamma / t$ obtained from the NH gap equation in Eq. (5) at $\beta \rightarrow \infty$ and $\mu=0$ for (a) $U_{1} / t=1.8$ and (b) $U_{1} / t=10$. We assume a cubic lattice with energy dispersion $\epsilon_{k}=-2 t\left(\cos k_{x}+\cos k_{y}+\cos k_{z}\right)$, where $t$ is the hopping amplitude. The dashed lines denote the asymptotic behavior in the strong-dissipation limit. The insets show (a) an enlarged view near the origin (weak dissipation) and (b) that of the real part. 
localization due to the QZE facilitates formation of on site molecules of fermions for strong dissipation and consequently superfluidity reappears. In fact, the solution of the gap equation approaches $\Delta_{0}=U / 2$ in the strongdissipation limit, as shown by the dashed lines in Fig. 1. This is consistent with the fact that the physics is dominated by the on-site interaction under the QZE, supporting our mean-field analysis. On the other hand, under strong attraction, fermions form bosonic molecules almost at single sites and thus the molecules can survive under dissipation. In this case, the effect of dissipation is to give rise to an effective one-body loss of molecules and the remaining molecules can undergo Bose-Einstein condensation.

We here point out that the breakdown and restoration of superfluidity present clear signatures of the emergence of exceptional points, where the Hamiltonian cannot be diagonalized [51,52]. In fact, when $\operatorname{Re} \Delta_{0}=0$, the meanfield Hamiltonian $H_{\mathrm{MF}}$ cannot be diagonalized for $\xi_{\boldsymbol{k}}= \pm \operatorname{Im} \Delta_{0}$. Figure 2(a) and 2(b) show the energy spectrum of quasiparticles in two dimensions. The regions where the orange and blue surfaces merge form exceptional points, lines [Fig. 2(c)], and surfaces [Fig. 2(d)] in one-, two-, and three-dimensional systems, respectively. Such characteristic behavior has its origin in a parity-particlehole $(C P)$ symmetry $C P H_{\mathrm{MF}}(\boldsymbol{k})(C P)^{-1}=-H_{\mathrm{MF}}(\boldsymbol{k})$ of the mean-field Hamiltonian $H_{\mathrm{MF}}(\boldsymbol{k})=\xi_{\boldsymbol{k}} \sigma_{z}+i \operatorname{Im} \Delta_{0} \sigma_{x}$, where $C P=\sigma_{x} K, \sigma_{x, z}$ are the Pauli matrices, and $K$ is complex conjugation [72-75]. In fact, as a function of the momentum, $H_{\mathrm{MF}}(\boldsymbol{k})$ exhibits spontaneous breaking of the $C P$ symmetry at the exceptional points, whose dimensionality is indeed protected by the symmetry constraint [59]. Thus, (a)

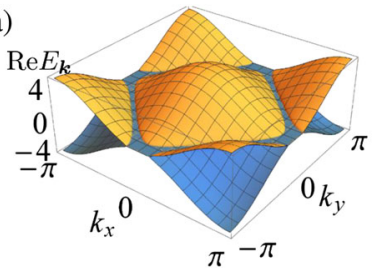

(c)

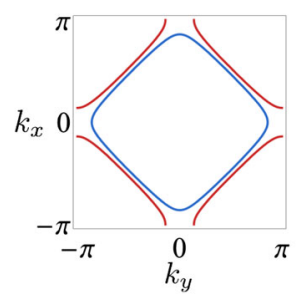

(b)

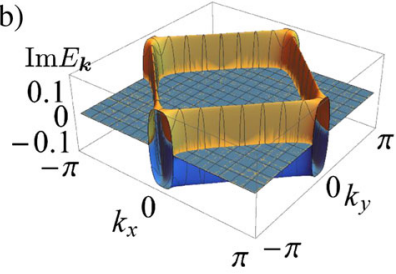

(d)

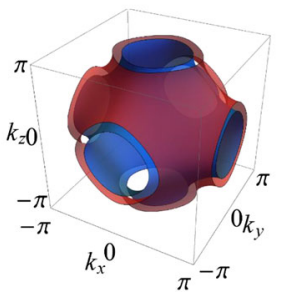

FIG. 2. (a) Real and (b) imaginary parts of the quasiparticle energy spectrum $E_{k}= \pm \sqrt{\epsilon_{k}^{2}+\Delta_{0}^{2}}$ (orange for the positive sign, blue for the negative sign) at the critical points. (c) Exceptional lines in two dimensions. (d) Exceptional surfaces in three dimensions. The energy dispersion is for a square lattice $\epsilon_{\boldsymbol{k}}=$ $-2\left(\cos k_{x}+\cos k_{y}\right)$ in (a), (b), and (c), and for a cubic lattice $\epsilon_{\boldsymbol{k}}=-2\left(\cos k_{x}+\cos k_{y}+\cos k_{z}\right)$ in (d). The gap is set to $\Delta_{0}=$ $0.19 i$ for (a), (b), and (c), and $\Delta_{0}=0.4 i$ for (d). the quantum phase transitions of the NH superfluid cannot be classified into the conventional first- or second-order phase transitions in Hermitian systems, but are attributed to the emergence of exceptional points unique to nonHermiticity. We note here that the two exceptional points corresponding to the breakdown and restoration of superfluidity merge and disappear as the strength of attraction increases. Intriguingly, as a remnant of the merged exceptional points, the real part of the superfluid gap for intermediate strengths of $U_{1}$ shows a characteristic minimum at a certain strength of dissipation [59]; the gap is first suppressed by dissipation, but enhanced again by the QZE as the dissipation is further increased.

Furthermore, the emergence of exceptional manifolds leads to some intriguing dynamics in the $\mathrm{NH}$ superfluid. In Fig. 2(b), the imaginary part of the quasiparticle energy takes a positive finite value only in between the exceptional lines or surfaces, amplifying quasiparticle distribution in the particular region of the Brillouin zone through the time evolution. The characteristic structure in Fig. 2(b), which can be used as a smoking gun of the non-Hermiticity, can be observed as long as $\operatorname{Im} \Delta_{0}>0$ even in a region away from the breakdown and restoration points.

We note that the nontrivial solution of the $\mathrm{NH}$ gap equation may give a metastable superfluid, which corresponds to a local minimum of the real part of the energy. Whether the superfluid is metastable or not is decided from comparison of ground-state energies between the superfluid state and the normal state, as detailed in the Supplemental Material [59].

From these results, we obtain a phase diagram of the $\mathrm{NH}$ BCS model as shown in Fig. 3. In the blue region, the superfluid state is an effective ground state of the NH BCS Hamiltonian. When the dissipation is increased, the superfluid state remains stable if the attraction is sufficiently strong. When the system enters the red region, the superfluid state becomes metastable with respect to the real part

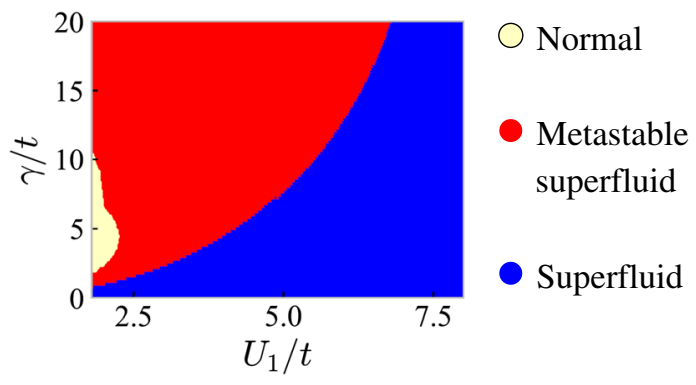

FIG. 3. Phase diagram of the NH BCS model at $\beta \rightarrow \infty$ and $\mu=0$. The yellow region corresponds to the normal phase. The red region shows the metastable superfluid phase for which a non-trivial solution of the gap equation gives a local energy minimum. The blue region shows the superfluid phase corresponding to a nontrivial solution of the gap equation that gives an effective ground state. A region with small $U_{1}$ is not shown because of the limitation of numerical calculations. 
of the energy. The metastable superfluid undergoes an unconventional quantum phase transition due to exceptional points in the case of weak attractions, leading to the disappearance of the superfluid state in the yellow region. Here we note that a similar phase diagram is obtained from an exact solution of a one-dimensional NH Hubbard model [76]. Although a similar formulation to obtain the gap equation can be made for a continuum system, the reentrant superfluidity is unique to the lattice system since the localization due to the QZE cannot occur without a lattice. On the other hand, the breakdown of the superfluidity and the related phase transitions can occur in the continuum system.

Towards experimental realization.-The NH quantum phase transitions can be observed by controlling a twobody loss rate. Since the superfluid is metastable in the red region, it can be realized by slowly increasing dissipation from the blue region. For weak attraction $U_{1}$, the superfluid undergoes an unconventional phase transition to the normal state due to the exceptional points. To observe the reappearance of the superfluidity under large $\gamma$, we may first prepare a metastable superfluid at large $U_{1}$ and $\gamma$, and then decrease $U_{1}$. Finally, the metastablity of the superfluid can be confirmed through comparison of the results between fast and slow increases of the dissipation from $\gamma=0$. For a detailed discussion including the relevant timescales, see the Supplemental Material [59].

These NH superfluids are expected to be realized with ultracold atoms under inelastic collisions. For example, a superfluid of ${ }^{173} \mathrm{Yb}$ atoms with an orbital Feshbach resonance [43] offers one such candidate since it is inevitably accompanied by two-body losses as observed experimentally [47-49]. The effect of non-Hermiticity on the superfluid gap can be observed by spectroscopy with Raman transitions between hyperfine levels or a clock transition $[49,50]$. Furthermore, concerning the control of two-body loss rates, introducing dissipation with photoassociation techniques [70] may also enable the realization of $\mathrm{NH}$ superfluids with ${ }^{40} \mathrm{~K}$ and ${ }^{6} \mathrm{Li}$ [53-57]. Although the strength of dissipation is usually fixed by scattering properties of atoms, dissipation engineering using photoassociation techniques will be a feasible method for realizing the $\mathrm{NH}$ fermionic superfluidity.

Conclusions. - We have investigated how the BCS superfluidity is extended to $\mathrm{NH}$ quantum systems under inelastic interactions. We have elucidated some remarkable features unique to the $\mathrm{NH}$ fermionic superfluidity, such as exotic Bogoliubov quasiparticles which belong to neither fermions nor bosons and found unconventional quantum phase transitions unique to non-Hermiticity. In particular, for weak attraction, it has been revealed that the superfluidity breaks down with increasing dissipation but shows reentrant behavior as dissipation is further increased. Remarkably, these phase transitions are accompanied by distinctive features of the non-Hermiticity, i.e., the emergence of exceptional points, lines, and surfaces in the quasiparticle Hamiltonian for one, two, and three dimensions. These characteristic features will play a decisive role in detecting $\mathrm{NH}$ phase transitions in experiments. On the other hand, for strong attraction, the superfluid state is not suppressed but enhanced due to the confinement of molecules to single sites via the QZE. While we have focused on a conventional $s$-wave superfluid, $p$-wave, $d$-wave, and other exotic superfluids in $\mathrm{NH}$ systems will also be relevant for experiments, and merit future investigation.

We are grateful to Yuto Ashida, Shunsuke Furukawa, Tsuneya Yoshida, and Yoshiro Takahashi for fruitful discussions. This work was supported by KAKENHI (Grants No. JP16J05078, No. JP16K05501, No. JP17J03883, No. JP18H01140, No. JP18H01145, and No. JP19H01838) and a Grant-in-Aid for Scientific Research on Innovative Areas (KAKENHI Grant No. JP15H05855) from the Japan Society for the Promotion of Science. M. N. was supported by RIKEN Special Postdoctoral Researcher Program. K. A. and K. T. thank JSPS for support from a Research Fellowship for Young Scientists. K. Y. is supported by Doctoral Program for World-leading Innovative \& Smart Education, Ministry of Education, Culture, Sports, Science and Technology.

"yamamoto.kazuki.72n@st.kyoto-u.ac.jp

[1] C. M. Bender and S. Boettcher, Phys. Rev. Lett. 80, 5243 (1998).

[2] C. M. Bender, Rep. Prog. Phys. 70, 947 (2007).

[3] M. Nakagawa, N. Kawakami, and M. Ueda, Phys. Rev. Lett. 121, 203001 (2018).

[4] J. A. S. Lourenço, R. L. Eneias, and R. G. Pereira, Phys. Rev. B 98, 085126 (2018).

[5] Y. Ashida, S. Furukawa, and M. Ueda, Phys. Rev. A 94, 053615 (2016).

[6] Y. Ashida, S. Furukawa, and M. Ueda, Nat. Commun. 8, 15791 (2017).

[7] K. Kawabata, Y. Ashida, and M. Ueda, Phys. Rev. Lett. 119, 190401 (2017).

[8] M. S. Rudner and L. S. Levitov, Phys. Rev. Lett. 102, 065703 (2009).

[9] T. E. Lee, Phys. Rev. Lett. 116, 133903 (2016).

[10] H. Shen, B. Zhen, and L. Fu, Phys. Rev. Lett. 120, 146402 (2018).

[11] F. K. Kunst, E. Edvardsson, J. C. Budich, and E. J. Bergholtz, Phys. Rev. Lett. 121, 026808 (2018).

[12] S. Yao, F. Song, and Z. Wang, Phys. Rev. Lett. 121, 136802 (2018).

[13] K. Kawabata, K. Shiozaki, and M. Ueda, Phys. Rev. B 98, 165148 (2018).

[14] Z. Gong, Y. Ashida, K. Kawabata, K. Takasan, S. Higashikawa, and M. Ueda, Phys. Rev. X 8, 031079 (2018).

[15] K. Kawabata, S. Higashikawa, Z. Gong, Y. Ashida, and M. Ueda, Nat. Commun. 10, 297 (2019). 
[16] K. Kawabata, K. Shiozaki, M. Ueda, and M. Sato, arXiv: 1812.09133.

[17] H. Zhou and J. Y. Lee, Phys. Rev. B 99, 235112 (2019).

[18] T. E. Lee and C.-K. Chan, Phys. Rev. X 4, 041001 (2014).

[19] A. Ghatak and T. Das, Phys. Rev. B 97, 014512 (2018).

[20] L. Zhou and X. Cui, iScience 14, 257 (2019).

[21] N. M. Chtchelkatchev, A. A. Golubov, T. I. Baturina, and V. M. Vinokur, Phys. Rev. Lett. 109, 150405 (2012).

[22] A. Guo, G. J. Salamo, D. Duchesne, R. Morandotti, M. Volatier-Ravat, V. Aimez, G. A. Siviloglou, and D. N. Christodoulides, Phys. Rev. Lett. 103, 093902 (2009).

[23] C. E. Rüter, K. G. Makris, R. El-Ganainy, D. N. Christodoulides, M. Segev, and D. Kip, Nat. Phys. 6, 192 (2010).

[24] L. Feng, M. Ayache, J. Huang, Y.-L. Xu, M.-H. Lu, Y.-F. Chen, Y. Fainman, and A. Scherer, Science 333, 729 (2011).

[25] A. Regensburger, C. Bersch, M.-A. Miri, G. Onishchukov, D. N. Christodoulides, and U. Peschel, Nature (London) 488, 167 (2012).

[26] J. M. Zeuner, M. C. Rechtsman, Y. Plotnik, Y. Lumer, S. Nolte, M. S. Rudner, M. Segev, and A. Szameit, Phys. Rev. Lett. 115, 040402 (2015).

[27] J. Li, A. K. Harter, J. Liu, L. de Melo, Y. N. Joglekar, and L. Luo, Nat. Commun. 10, 855 (2019).

[28] L. Xiao, X. Zhan, Z. H. Bian, K. K. Wang, X. Zhang, X. P. Wang, J. Li, K. Mochizuki, D. Kim, N. Kawakami, W. Yi, H. Obuse, B. C. Sanders, and P. Xue, Nat. Phys. 13, 1117 (2017).

[29] H. Zhou, C. Peng, Y. Yoon, C. W. Hsu, K. A. Nelson, L. Fu, J. D. Joannopoulos, M. Soljačić, and B. Zhen, Science 359, 1009 (2018).

[30] L. Xiao, K. Wang, X. Zhan, Z. Bian, K. Kawabata, M. Ueda, W. Yi, and P. Xue,arXiv:1812.01213.

[31] S. Dürr, J. J. García-Ripoll, N. Syassen, D. M. Bauer, M. Lettner, J. I. Cirac, and G. Rempe, Phys. Rev. A 79, 023614 (2009).

[32] J. J. Garca-Ripoll, S. Dürr, N. Syassen, D. M. Bauer, M. Lettner, G. Rempe, and J. I. Cirac, New J. Phys. 11, 013053 (2009).

[33] A. J. Daley, J. M. Taylor, S. Diehl, M. Baranov, and P. Zoller, Phys. Rev. Lett. 102, 040402 (2009).

[34] A. Kantian, M. Dalmonte, S. Diehl, W. Hofstetter, P. Zoller, and A. J. Daley, Phys. Rev. Lett. 103, 240401 (2009).

[35] T. Yoshida, R. Peters, and N. Kawakami, Phys. Rev. B 98, 035141 (2018).

[36] S. Yao and Z. Wang, Phys. Rev. Lett. 121, 086803 (2018).

[37] A. J. Daley, Adv. Phys. 63, 77 (2014).

[38] J. Dalibard, Y. Castin, and K. Mølmer, Phys. Rev. Lett. 68, 580 (1992).

[39] J. Bardeen, L. N. Cooper, and J. R. Schrieffer, Phys. Rev. 108, 1175 (1957).

[40] C. A. Regal, M. Greiner, and D. S. Jin, Phys. Rev. Lett. 92, 040403 (2004).

[41] M. W. Zwierlein, C. A. Stan, C. H. Schunck, S. M. F. Raupach, A. J. Kerman, and W. Ketterle, Phys. Rev. Lett. 92, 120403 (2004).

[42] M. Bartenstein, A. Altmeyer, S. Riedl, S. Jochim, C. Chin, J. H. Denschlag, and R. Grimm, Phys. Rev. Lett. 92, 120401 (2004).
[43] R. Zhang, Y. Cheng, H. Zhai, and P. Zhang, Phys. Rev. Lett. 115, 135301 (2015).

[44] M. Iskin, Phys. Rev. A 94, 011604(R) (2016).

[45] J. Xu, R. Zhang, Y. Cheng, P. Zhang, R. Qi, and H. Zhai, Phys. Rev. A 94, 033609 (2016).

[46] L. He, J. Wang, S.-G. Peng, X.-J. Liu, and H. Hu, Phys. Rev. A 94, 043624 (2016).

[47] M. Höfer, L. Riegger, F. Scazza, C. Hofrichter, D. R. Fernandes, M. M. Parish, J. Levinsen, I. Bloch, and S. Fölling, Phys. Rev. Lett. 115, 265302 (2015).

[48] G. Pagano, M. Mancini, G. Cappellini, L. Livi, C. Sias, J. Catani, M. Inguscio, and L. Fallani, Phys. Rev. Lett. 115, 265301 (2015).

[49] G. Cappellini, L. F. Livi, L. Franchi, D. Tusi, D. Benedicto Orenes, M. Inguscio, J. Catani, and L. Fallani, Phys. Rev. X 9, 011028 (2019).

[50] N. Darkwah Oppong, L. Riegger, O. Bettermann, M. Höfer, J. Levinsen, M. M. Parish, I. Bloch, and S. Fölling, Phys. Rev. Lett. 122, 193604 (2019).

[51] W. D. Heiss, J. Phys. A 45, 444016 (2012).

[52] M. V. Berry, Czech. J. Phys. 54, 1039 (2004).

[53] J. Stewart, J. Gaebler, and D. Jin, Nature (London) 454, 744 (2008).

[54] C. Chin, M. Bartenstein, A. Altmeyer, S. Riedl, S. Jochim, J. H. Denschlag, and R. Grimm, Science 305, 1128 (2004).

[55] J. K. Chin, D. Miller, Y. Liu, C. Stan, W. Setiawan, C. Sanner, K. Xu, and W. Ketterle, Nature (London) 443, 961 (2006).

[56] T. Stöferle, H. Moritz, K. Günter, M. Köhl, and T. Esslinger, Phys. Rev. Lett. 96, 030401 (2006).

[57] D. Mitra, P. T. Brown, E. Guardado-Sanchez, S. S. Kondov, T. Devakul, D. A. Huse, P. Schauss, and W. S. Bakr, Nat. Phys. 14, 173 (2018).

[58] D. C. Brody, J. Phys. A 47, 035305 (2014).

[59] See Supplemental Material at http://link.aps.org/ supplemental/10.1103/PhysRevLett.123.123601, which includes Refs. [60,61], for the detailed calculations in the $\mathrm{NH}$ mean-field theory of fermionic superfluidity, the chemical potential in non-Hermitian systems, the calculation of the condensation energy of the superfluid state, additional numerical results in the intermediate regime $U_{1} / t=2.5$, a physical origin of exceptional points due to spontaneous $C P$ symmetry breaking, and details of experimental setups for probing the non-Hermitian superfluid.

[60] A. J. Leggett and F. Sols, Found. Phys. 21, 353 (1991).

[61] A. J. Leggett, Quantum Liquids: Bose Condensation and Cooper Pairing in Condensed-Matter Systems (Oxford University Press, Oxford, 2006).

[62] A. J. Leggett, J. Phys. (Paris), Colloq. 41, C7 (1980).

[63] A. J. Leggett, in Modern Trends in the Theory of Condensed Matter (Springer, Berlin, 1980), pp. 13-27.

[64] P. Nozières and S. Schmitt-Rink, J. Low Temp. Phys. 59, 195 (1985).

[65] R. B. Diener, R. Sensarma, and M. Randeria, Phys. Rev. A 77, 023626 (2008).

[66] N. Syassen, D. M. Bauer, M. Lettner, T. Volz, D. Dietze, J. J. García-Ripoll, J. I. Cirac, G. Rempe, and S. Dürr, Science 320, 1329 (2008). 
[67] M. J. Mark, E. Haller, K. Lauber, J. G. Danzl, A. Janisch, H. P. Büchler, A. J. Daley, and H.-C. Nägerl, Phys. Rev. Lett. 108, 215302 (2012).

[68] G. Barontini, R. Labouvie, F. Stubenrauch, A. Vogler, V. Guarrera, and H. Ott, Phys. Rev. Lett. 110, 035302 (2013).

[69] B. Zhu, B. Gadway, M. Foss-Feig, J. Schachenmayer, M. L. Wall, K. R. A. Hazzard, B. Yan, S. A. Moses, J. P. Covey, D. S. Jin, J. Ye, M. Holland, and A. M. Rey, Phys. Rev. Lett. 112, 070404 (2014).

[70] T. Tomita, S. Nakajima, I. Danshita, Y. Takasu, and Y. Takahashi, Sci. Adv. 3, e1701513 (2017).
[71] B. Yan, S. A. Moses, B. Gadway, J. P. Covey, K. R. Hazzard, A. M. Rey, D. S. Jin, and J. Ye, Nature (London) 501, 521 (2013).

[72] T. Yoshida, R. Peters, N. Kawakami, and Y. Hatsugai, Phys. Rev. B 99, 121101(R) (2019).

[73] J. C. Budich, J. Carlström, F. K. Kunst, and E. J. Bergholtz, Phys. Rev. B 99, 041406(R) (2019).

[74] R. Okugawa and T. Yokoyama, Phys. Rev. B 99, 041202(R) (2019).

[75] H. Zhou, J. Y. Lee, S. Liu, and B. Zhen, Optica 6, 190 (2019).

[76] M. Nakagawa, N. Kawakami, and M. Ueda (to be published). 